\title{
Teaching NeuroImages: Intracranial malignant triton tumor
}

\author{
An uncommon location of a rare tumor
}

Adriano Basso Dias, MD, Eduardo Cambruzzi, PhD, Cláudia Scherber Giugno, MD, Rodrigo Miranda de Curtis, MD, Lázaro Luís Faria do Amaral, MD, and Rene Lenhardt, MD

Neurology ${ }^{\circledR}$ 2019;92:e1401-e1402. doi:10.1212/WNL.0000000000007144
Correspondence

Dr. Lenhardt

reneneuro@gmail.com

Figure 1 Brain MRI

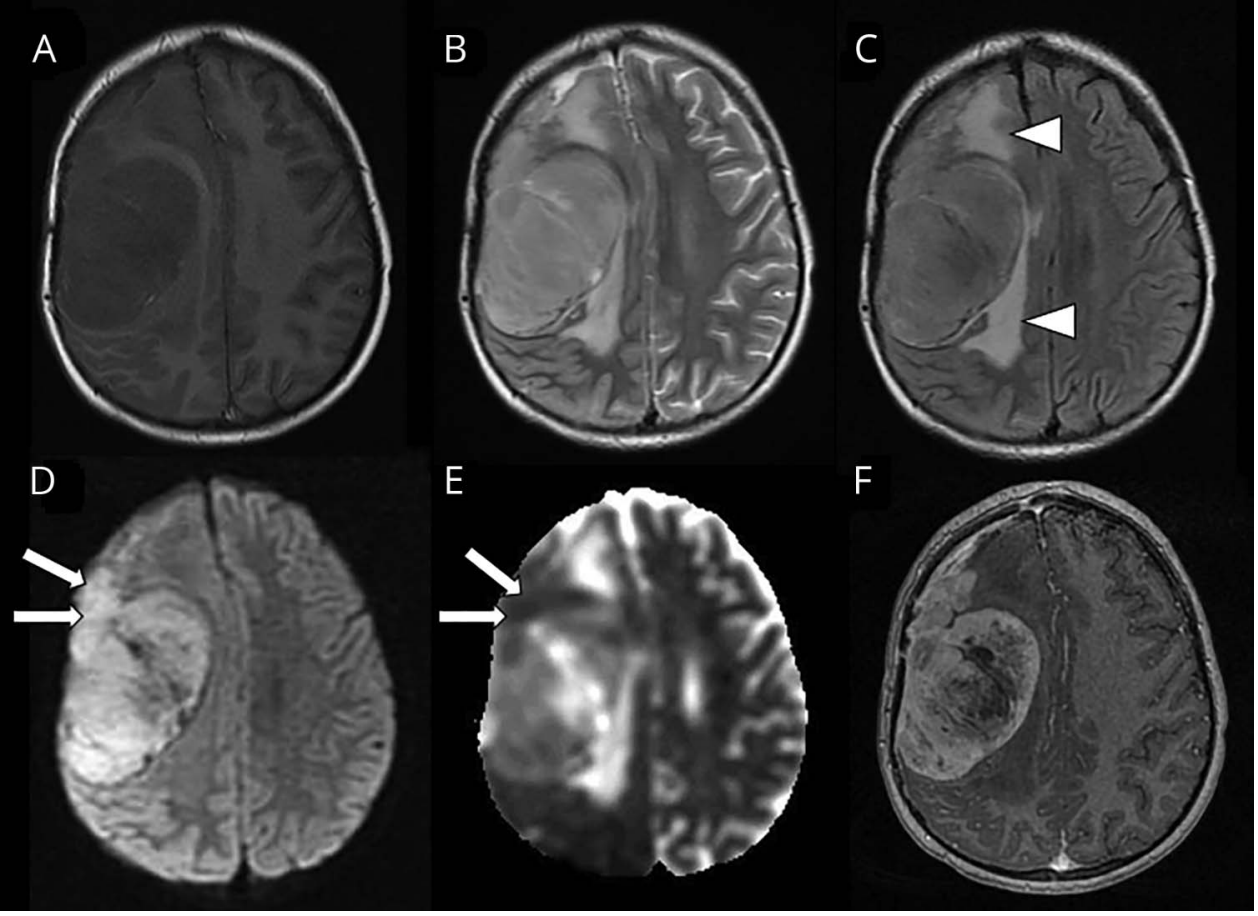

Axial brain MRI reveals a large extraaxial right frontal mass, hypointense on T1-weighted imaging (A) and hyperintense on T2-weighted imaging (B), associated with moderate surrounding vasogenic edema (arrowheads), better seen on fluidattenuated inversion recovery (C). The lesion demonstrated areas of diffusion restriction (arrows) on diffusion-weighted imaging (D) and apparent diffusion coefficient $(E)$ and heterogeneous enhancement on postcontrast T1-weighted imaging (F).
A 5-year-old girl with neurofibromatosis type 1 (NF1) presented with a 1-month history of progressive left hemiparesis. Brain MRI revealed a large extra-axial right frontal mass (figure 1). The pathologic findings after complete resection were compatible with malignant triton tumor (MTT) (figure 2). MTT is an aggressive subtype of malignant peripheral nerve sheath tumor, histopathologically characterized by a high-grade spindle cell tumor, with immunohistochemistry demonstrating reaction to skeletal muscle stains, such as desmin and myogenin. ${ }^{1,2}$ MTT is associated with NF1 in $38 \%$ of cases. ${ }^{1}$ Intracranial MTT is exceedingly rare, usually presenting radiologically as a rapidly enlarging, heterogeneous, and enhancing mass. ${ }^{2}$

\section{MORE ONLINE}

\section{$\rightarrow$ Teaching slides}

links.lww.com/WNL/ A838

\section{Study funding}

No targeted funding reported. 


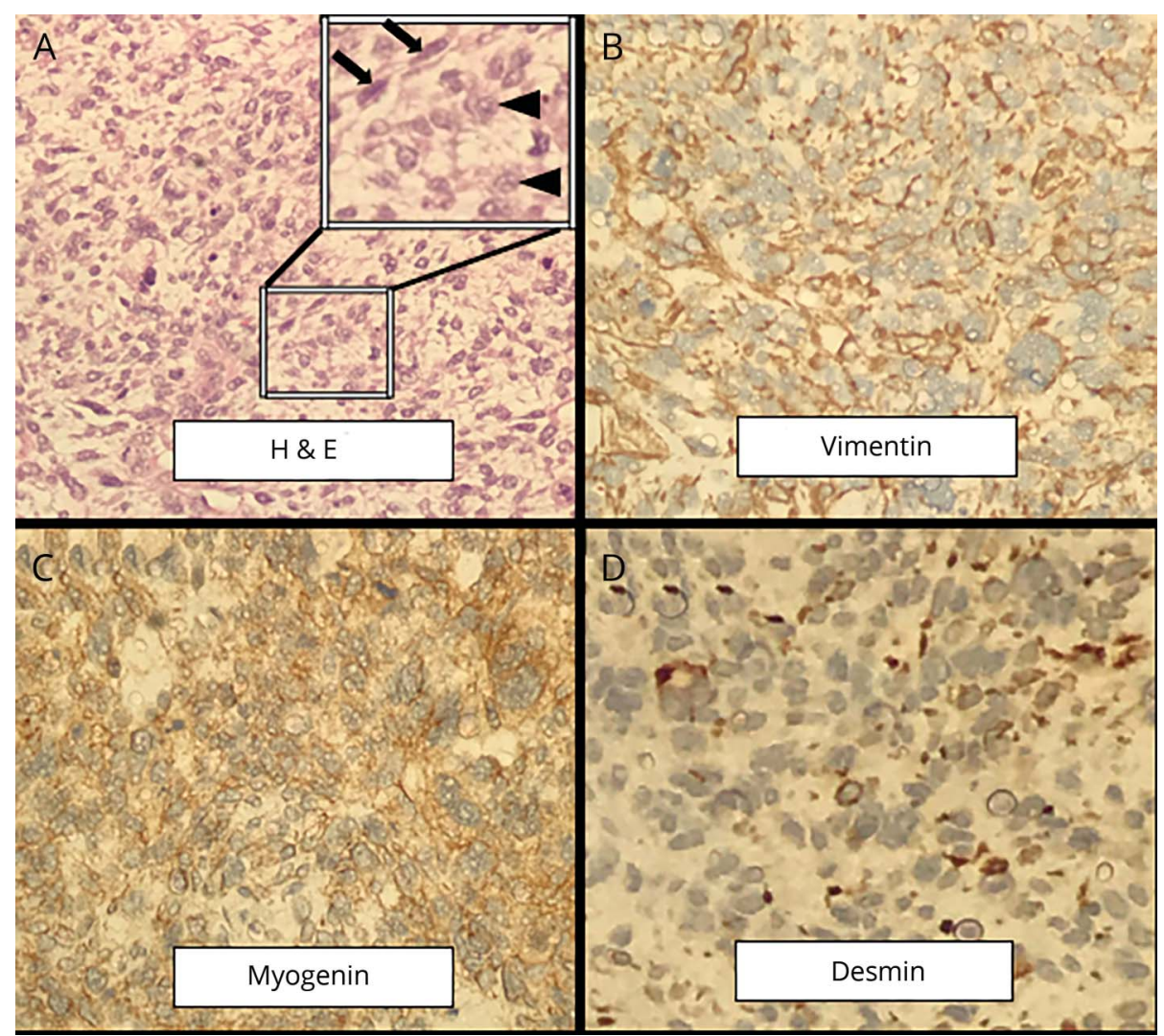

(A) Histologic evaluation (hematoxylin \& eosin, $\times 200$ ) shows a neoplastic process composed of spindle cells showing a fascicular growth pattern (arrows), extensive pleomorphism, and high mitotic index (arrowheads). Immunohistochemical analysis $(\times 200)$ demonstrates positive immunoexpression for vimentin (B), myogenin (C), and desmin (D), reflecting areas of rhabdomyoblastic differentiation.

\section{Disclosure}

The authors report no disclosures relevant to the manuscript. Go to Neurology.org/N for full disclosures.

\section{Appendix Authors}

\begin{tabular}{llll}
\hline Name & Location & Role & Contribution \\
\hline $\begin{array}{l}\text { Adriano } \\
\text { Basso Dias, } \\
\text { MD }\end{array}$ & $\begin{array}{l}\text { Irmandade Santa } \\
\text { Casa de } \\
\text { Misericórdia de } \\
\text { Porto Alegre, Brazil }\end{array}$ & Author & $\begin{array}{l}\text { Study concept and } \\
\text { manuscript writing }\end{array}$ \\
\hline $\begin{array}{l}\text { Eduardo } \\
\text { Cambruzzi, } \\
\text { PhD }\end{array}$ & $\begin{array}{l}\text { Irmandade Santa } \\
\text { Casa de }\end{array}$ & Author & Major role in the \\
& $\begin{array}{l}\text { Misericórdia de } \\
\text { Porto Alegre, Brazil }\end{array}$ & & acquisition of data \\
$\begin{array}{l}\text { Cláudia } \\
\text { Scherber } \\
\text { Giugno, MD }\end{array}$ & $\begin{array}{l}\text { Irmandade Santa } \\
\text { Casa de } \\
\text { Misericórdia de }\end{array}$ & Author & $\begin{array}{l}\text { Major role in the } \\
\text { acquisition of data }\end{array}$ \\
\hline $\begin{array}{l}\text { Porto Alegre, Brazil } \\
\text { Miranda de } \\
\text { Curtis, MD }\end{array}$ & $\begin{array}{l}\text { Casa de } \\
\text { Misericórdia de } \\
\text { Porto Alegre, Brazil }\end{array}$ & Author & $\begin{array}{l}\text { Critical revision of the } \\
\text { manuscript for } \\
\text { important intellectual }\end{array}$ \\
\hline & & & content \\
\hline
\end{tabular}

Appendix (continued)

\begin{tabular}{|c|c|c|c|}
\hline Name & Location & Role & Contribution \\
\hline $\begin{array}{l}\text { Lázaro Luís } \\
\text { Faria do } \\
\text { Amaral, MD }\end{array}$ & $\begin{array}{l}\text { Beneficência } \\
\text { Portuguesa de São } \\
\text { Paulo, Brazil }\end{array}$ & Author & $\begin{array}{l}\text { Critical revision of the } \\
\text { manuscript for } \\
\text { important intellectual } \\
\text { content }\end{array}$ \\
\hline $\begin{array}{l}\text { Rene } \\
\text { Lenhardt, } \\
\text { MD }\end{array}$ & $\begin{array}{l}\text { Irmandade Santa } \\
\text { Casa de } \\
\text { Misericórdia de } \\
\text { Porto Alegre, Brazil }\end{array}$ & Author & $\begin{array}{l}\text { Critical revision of the } \\
\text { manuscript for } \\
\text { important intellectual } \\
\text { content, study } \\
\text { supervision }\end{array}$ \\
\hline
\end{tabular}

\section{References}

1. McConnell YJ, Giacomantonio CA. Malignant triton tumors: complete surgical resection and adjuvant radiotherapy associated with improved survival. J Surg Oncol 2012,106:51-56.

2. Smith RE, Kebriaei MA, Gard AP, Mccomb RD, Bridge JA, Lennarson PJ. Intracranial malignant triton tumor in a patient with neurofibromatosis type 1: case report and review of the literature. Brain Tumor Pathol 2014; 31:149-154. 


\section{Neurology}

\section{Teaching NeuroImages: Intracranial malignant triton tumor: An uncommon location of a rare tumor}

Adriano Basso Dias, Eduardo Cambruzzi, Cláudia Scherber Giugno, et al.

Neurology 2019;92;e1401-e1402

DOI 10.1212/WNL.0000000000007144

\section{This information is current as of March 18, 2019}

\section{Updated Information \&} Services

References

Subspecialty Collections

Permissions \& Licensing

Reprints including high resolution figures, can be found at: http://n.neurology.org/content/92/12/e1401.full

This article cites 2 articles, 0 of which you can access for free at: http://n.neurology.org/content/92/12/e1401.full\#ref-list-1

This article, along with others on similar topics, appears in the following collection(s):

MRI

http://n.neurology.org/cgi/collection/mri

Nerve tumor

http://n.neurology.org/cgi/collection/nerve_tumor

Neurofibromatosis

http://n.neurology.org/cgi/collection/neurofibromatosis

Information about reproducing this article in parts (figures,tables) or in its entirety can be found online at:

http://www.neurology.org/about/about_the_journal\#permissions

Information about ordering reprints can be found online:

http://n.neurology.org/subscribers/advertise

Neurology ${ }^{\circledR}$ is the official journal of the American Academy of Neurology. Published continuously since 1951, it is now a weekly with 48 issues per year. Copyright (O 2019 American Academy of Neurology. All rights reserved. Print ISSN: 0028-3878. Online ISSN: 1526-632X.

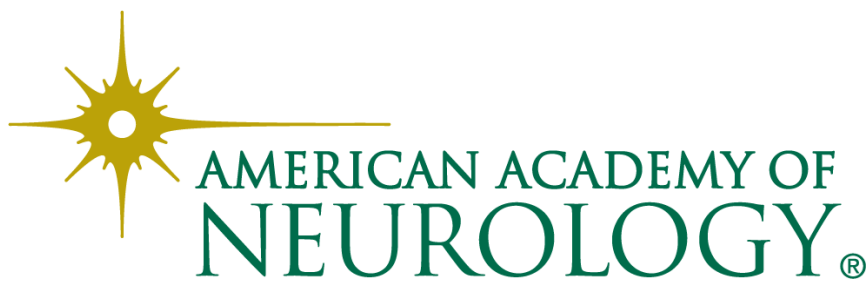

